\title{
Atomic corrugation in scanning tunneling microscopy images of the $\mathrm{Fe}(001)-p(1 \times 1) \mathrm{O}$ surface
}

\author{
A. Picone,${ }^{1}$ G. Fratesi, ${ }^{2}$ A. Brambilla,,${ }^{1}$, P. Sessi, ${ }^{1}$ F. Donati, ${ }^{3}$ S. Achilli, ${ }^{2}$ L. Maini, ${ }^{2}$ M. I. Trioni,${ }^{4}$ C. S. Casari, ${ }^{3,5}$ \\ M. Passoni, ${ }^{3}$ A. Li Bassi, ${ }^{3,5}$ M. Finazzi, ${ }^{1}$ L. Duò, ${ }^{1}$ and F. Ciccacci ${ }^{1}$ \\ ${ }^{1}$ CNISM, NEMAS, and Dipartimento di Fisica, Politecnico di Milano, Piazza Leonardo da Vinci 32, I-20133 Milano, Italy \\ ${ }^{2}$ Dipartimento di Scienza dei Materiali, Università di Milano-Bicocca, Via Cozzi 53, 20125 Milano, Italy \\ ${ }^{3}$ CNISM, NEMAS, and Dipartimento di Energia, Politecnico di Milano, Via Ponzio 34/3, I-20133 Milano, Italy \\ ${ }^{4}$ CNR-INFM, UdR Milano-Bicocca, Via Cozzi 53, 20125 Milano, Italy \\ ${ }^{5}$ Center for Nano Science and Technology of IIT@PoliMI, Via Pascoli 70/3, I-20133 Milano, Italy \\ (Received 20 November 2009; revised manuscript received 5 February 2010; published 25 March 2010)
}

\begin{abstract}
We have investigated the formation of scanning tunneling microscopy (STM) atomically resolved images of the $\mathrm{Fe}(001)-p(1 \times 1) \mathrm{O}$ surface. The latter is characterized by a high in-plane symmetry for both oxygen and iron atoms, thus representing a very appealing template for understanding how to distinguish between oxygen and metal atoms in STM images of an oxidized metal surface. We report on the occurrence of a corrugation reversal as a function of the tip-sample distance, and we use the conclusions of such an investigation for interpreting the experimental images of an oxygen vacancy created on the surface. Our experimental work, supported by ab initio density-functional theory calculations, allows us to assess the fundamental role of the local potential barrier in determining the STM image formation.
\end{abstract}

DOI: 10.1103/PhysRevB.81.115450

PACS number(s): 68.37.Ef, 68.43.Bc, 73.20.At

\section{INTRODUCTION}

The surfaces of metal oxides and the interfaces between metal and oxide thin films are fundamental components of layered magnetic structures, ${ }^{1}$ with promising implications on the realization of future magnetic nanodevices. In order to explore the properties of such systems at atomic scales, suitable experimental and theoretical approaches are needed. From the experimental point of view, the scanning tunneling microscopy (STM) is one of the most significant techniques in this sense. As an example, the understanding of the mechanisms that drive the oxidation processes of metal surfaces at the atomic scale can also require the identification of the active sites for reactions. ${ }^{2,3}$ The latter are often related to surface defects such as vacancies and adsorbed atoms or molecules. ${ }^{4,5}$ It is therefore important to be able to precisely interpret the atomically resolved features observed in the STM images. This means, for instance, assigning such features, as seen on the oxidized metal surface, either to metal or oxygen atoms. This task can be extremely challenging, as both surface geometry and electronic structure compete in the formation of the STM topographic profile. This competition, and in some cases also the influence of the STM tip, is known to induce image inversion effects, so that $\mathrm{O}$ adsorbates can appear as either protrusions or depressions on account of the measurement conditions. ${ }^{6-9}$ In order to single out the role of each contribution in the formation of the STM image, a comparison with simulated images, calculated on the basis of suitable theoretical approaches, is eventually required.

In this paper we explore the topographical details, as seen by STM, of the $\mathrm{Fe}(001)-p(1 \times 1) \mathrm{O}$ surface. This system is obtained through the well ordered chemisorption of a single atomic layer of oxygen on the $\mathrm{Fe}(001)$ surface. The adsorbed $\mathrm{O}$ atoms settle in the fourfold symmetric hollow sites of the surface square cells, standing in a higher position with respect to the first Fe layer. ${ }^{10}$ This symmetrical arrangement is accompanied by an adsorption induced surface relaxation. This leads to an equilibrium geometry characterized by an expansion of the first interlayer spacing of the $\mathrm{Fe}(001)$ substrate of about $10 \% .{ }^{11,12}$ On this surface, both oxygen and iron atoms are distributed with the same in-plane symmetry, so that the identification of the atomically resolved features by STM cannot be straightforward. This peculiar system can indeed represent a very interesting workbench for exploring the role that factors such as the local density of states at the surface, and the decaying behavior of the electronic wave functions toward the vacuum play, in competition with the bare geometrical structure, for the determination of the STM image formation.

We then focus our attention on one particular kind of defect seen on the $\mathrm{Fe}(001)-p(1 \times 1) \mathrm{O}$ surface, namely, an oxygen vacancy, and analyze its topographical features as imaged by the STM. We compare all of the STM experimental results with theoretical predictions based on firstprinciples density-functional theory (DFT) calculations of the surface electronic structure. Such predictions allow us to emphasize the role of the local potential barrier in determining the STM image formation.

\section{EXPERIMENTAL AND COMPUTATIONAL METHODS}

The pristine Fe(001) substrate is a Fe film (about $100 \mathrm{~nm}$ thick) epitaxially grown in ultrahigh vacuum (UHV) conditions on top of a $\mathrm{MgO}(001)$ substrate. ${ }^{13}$ The $\mathrm{Fe}(001)-p(1$ $\times 1) \mathrm{O}$ surface is then obtained by exposure to $30 \mathrm{~L}$ of pure $\mathrm{O}_{2}$, followed by a flash heating at $900 \mathrm{~K}$, as previously reported. ${ }^{14,15}$ The STM measurements have been performed using an Omicron Variable Temperature STM in a UHV chamber connected to the preparation system. STM images have been acquired at room temperature in constant current mode with home-made electrochemically etched W tips.

In performing first-principles simulations, the Fe surface has been modeled by a slab composed of five Fe layers and 
$\mathrm{O}$ atoms adsorbed on both sides, in a periodically repeated supercell. Atomic coordinates of all atoms but the inner three Fe layers (fixed at the bulk spacing) have been obtained via optimization of the total energy of the system. All the results have been obtained within the generalized gradient approximation for the exchange and correlation functional as proposed by Perdew, Burke, and Ernzerhof (GGA-PBE), ${ }^{16}$ taking into account the spin polarization. The plane-wave ultrasoft pseudopotential method ${ }^{17}$ was used as implemented in the PWSCF code of the Quantum-ESPRESSO distribution. ${ }^{18}$ The pseudopotentials have been derived from scalarrelativistic all-electron atomic calculations. $\mathrm{Fe}$ and $\mathrm{O}$ pseudopotentials include $3 d-4 s$ and $2 s-2 p$ valence electrons, respectively. Nonlinear core corrections are used for Fe. The wave functions have been expanded up to a kinetic energy cutoff of $30 \mathrm{Ry}$; the effective potential and the charge density up to $200 \mathrm{Ry}$. The surface Brillouin zone integration has been performed with the Monkhorst-Pack ${ }^{19}$ scheme, adopting $\mathbf{k}_{\|}$-point meshes equivalent to a $16 \times 16$ mesh in the irreducible surface unit cell of $\mathrm{Fe}(001)$.

Constant current STM images have eventually been simulated by calculating the Kohn-Sham (KS) local density of states in the energy interval between $1 \mathrm{eV}$ below the Fermi level $\left(E_{\mathrm{F}}\right)$ and $E_{\mathrm{F}},{ }^{20}$ and by evaluating a constant density surface in the vacuum region. ${ }^{21,22}$ A Gaussian spatial broadening of $1 \AA$ was finally applied to mimic finite experimental resolution. It must be noted that this approximation does not consider the perturbation induced on the surface by the STM tip nor its spectral structure.

\section{RESULTS}

Spectroscopic and topographic characterizations of the pristine $\mathrm{Fe}(001)$ surface have revealed that the electronic states close to $E_{\mathrm{F}}$ are dominated by a surface state located at $+0.17 \mathrm{eV}$ (Ref. 23) and that the surface corrugation is extremely small, with average values below 2 pm. ${ }^{8}$ Both situations change dramatically when considering the $\mathrm{Fe}(001)-p(1 \times 1) \mathrm{O}$ surface.

For what concerns the spectroscopic features we have shown, by using STS, that the surface states close to $E_{\mathrm{F}}$ are characterized by two features located at about $0.5 \mathrm{eV}$ below $E_{\mathrm{F}}$ and $0.9 \mathrm{eV}$ above $E_{\mathrm{F}}{ }^{24}$ The theoretical approach used in the present work provides equivalent results, in terms of $\mathbf{k}_{\|}$-integrated electronic properties, as our previous study (based on the embedding approach with LAPW basis sets, see Ref. 24), additionally allowing for structural relaxations and for the simulation of $\mathrm{O}$ vacancies in larger surface unit cells. The computed vertical expansion of the first iron-iron spacing of the $\mathrm{Fe}(001)-p(1 \times 1) \mathrm{O}$ surface results to be $\Delta d_{\mathrm{Fe}-\mathrm{Fe}}=15 \%$ of the bulk spacing, and the distance between the oxygen and surface iron planes amounts to $d_{\mathrm{O}-\mathrm{Fe}}$ $=0.44 \AA$; these values are practically identical to recent computed ones $\left(16 \%\right.$ and $0.45 \AA$, respectively $\left.{ }^{25}\right)$ and in much better agreement with experimental values $\left[\Delta d_{\mathrm{Fe}-\mathrm{Fe}}\right.$ $=8 \pm 3 \%$ (Ref. 11) and $11 \pm 3 \%,{ }^{12}$ and $d_{\mathrm{O}-\mathrm{Fe}}$ $=0.45 \pm 0.04 \AA$ (Ref. 11) $]$ than earlier calculations $\left[\Delta d_{\mathrm{Fe}-\mathrm{Fe}}\right.$ $=23 \%$ and $d_{\mathrm{O}-\mathrm{Fe}}=0.38 \AA$ (Ref. 26)].

From the point of view of topographic features, the average surface corrugation of the $\mathrm{Fe}(001)-p(1 \times 1) \mathrm{O}$ surface no-

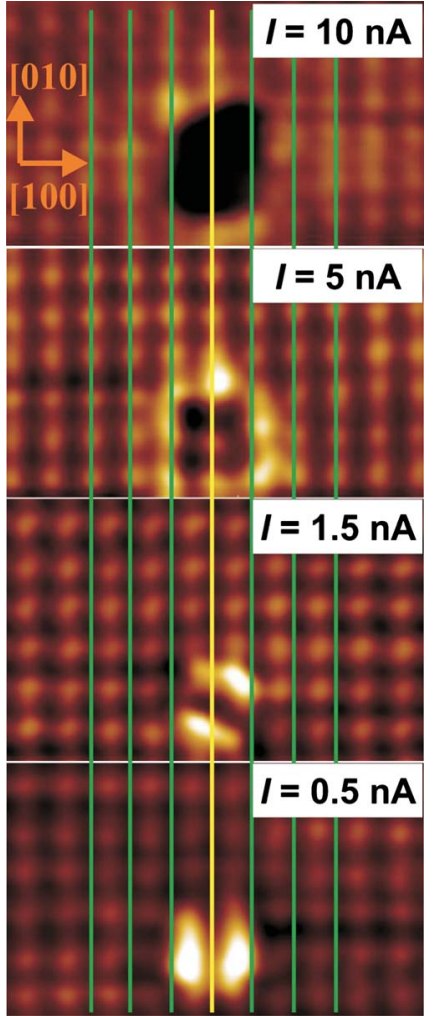

FIG. 1. (Color online) STM atomic resolution images of the $\mathrm{Fe}(001)-p(1 \times 1) \mathrm{O}$ surface. The sample bias is set at $V_{b}=0.1 \mathrm{~V}$ for every image, while the current set point is $0.5,1.5,5$, and $10 \mathrm{nA}$, from the lower to the upper image, respectively. The image area is about $2.9 \times 1.7 \mathrm{~nm}^{2}$ for each image. The vertical lines are guides to the eyes for highlighting the corrugation reversal.

ticeably increases, with respect to the clean Fe(001) surface, with average values up to $30 \mathrm{pm}$. Moreover we observe, for different $I-V$ set points, corresponding to different tip-sample distances, a corrugation reversal consisting of either oxygen atoms or iron atoms observed in the STM images, as described below.

\section{A. Evidence of a corrugation reversal}

In order to study a possible corrugation reversal as a function of the STM set points, it is essential to have a reference position, that can be located on a surface defect. The asgrown $\mathrm{Fe}(001)-p(1 \times 1) \mathrm{O}$ surface is usually very clean, flat (on a scale of tens of $\mathrm{nm}$ ) and free of defects. It has thus been necessary to induce some kind of defects on the surface. This has been done by applying a short (few seconds) $1.5 \mathrm{kV} \mathrm{Ar}^{+}$ ion sputtering pulse. One can expect that a defect will consist of either a simple oxygen vacancy or a more complicated structure, resulting from a local redistribution of the atoms.

Figure 1 reports atomic corrugation images of the $\mathrm{Fe}(001)-p(1 \times 1) \mathrm{O}$ surface taken at a constant sample bias $V_{b}=0.1 \mathrm{~V}$ and at different current set points, with the tunneling current $I$ equal to $0.5,1.5,5$, and $10 \mathrm{nA}$, from the lower to the upper image, respectively. The tip-sample distance varies correspondingly, from larger to smaller distances, with increasing current set points. We have estimated 


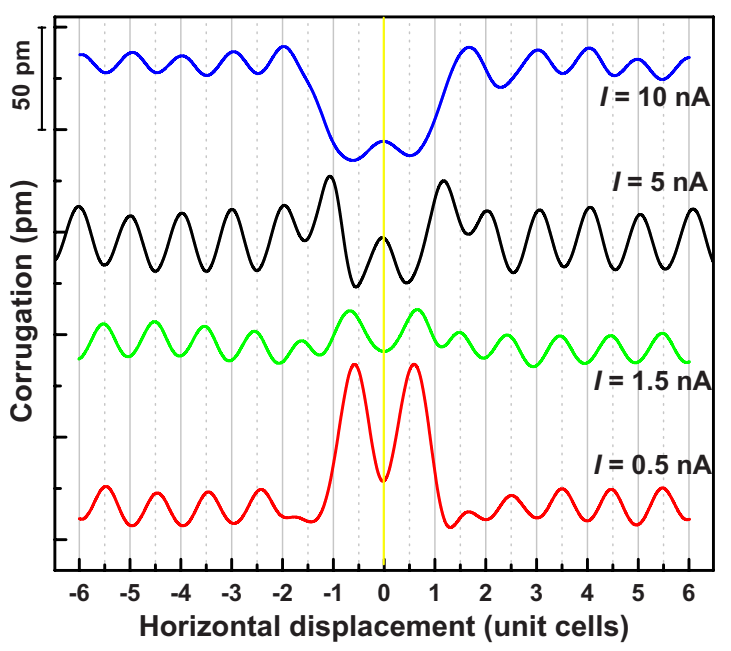

FIG. 2. (Color online) Horizontal line profiles of the STM images shown in Fig. 1. The lines pass through the center of the defect and are aligned making reference to such a feature (central vertical line in Fig. 1). One unit cell is equal to $0.29 \mathrm{~nm}$.

this distance as discussed in Ref. 24: it results to vary from about $5 \AA$ for $I=10 \mathrm{nA}$ to slightly less than $7 \AA$ for $I$ $=0.5 \mathrm{nA}$.

For each of the images reported in Fig. 1 we have drawn a horizontal line profile passing through the defect center (the horizontal lines are not shown in Fig. 1). The profiles are collected in Fig. 2, where the center of the defect has been chosen as the origin of the horizontal position for each profile. By inspecting Fig. 2 as regards displacements larger than 2 unit cells from the defect center, one has an immediate evidence of an inversion between peaks, corresponding to protrusions in the images, and valleys, corresponding to depressions, when comparing the $I=0.5 \mathrm{nA}$ and $I=1.5 \mathrm{nA}$ with the $I=5 \mathrm{nA}$ and $I=10 \mathrm{nA}$ cases, respectively. Such an inversion can be evidenced also by inspecting the images reported in Fig. 1, which have all been aligned along the [010] direction, by keeping the center of the defect on the same vertical line. The vertical lines can help to observe that rows of protrusions in the two upper images $(I=5 \mathrm{nA}$ and $I=10 \mathrm{nA})$ correspond to dim rows in the two lower images $(I=0.5 \mathrm{nA}$ and $I=1.5 \mathrm{nA})$. The known surface symmetry and unit cell eventually allow to identify this change in the atomic scale corrugation as either oxygen only or iron only atoms imaged in the two cases. This kind of corrugation reversal refers therefore to the distinct kind of atoms that are imaged by the STM with different experimental set points. A strong modification of the brightness levels is seen also on the defect itself. The topography of the defect will be discussed in Sec. III B.

We underline that the above results are not dependent on the bias condition, in particular on the sign of the tip-sample bias, provided its absolute value is small, typically below $100 \mathrm{meV}$. The use of larger biases generally resulted in less stable measurements with a worse resolution, so that they have not proved to be useful in determining the occurrence of a corrugation reversal

A first, semiquantitative analysis of the corrugation reversal mechanism can be based on the observation that a charge

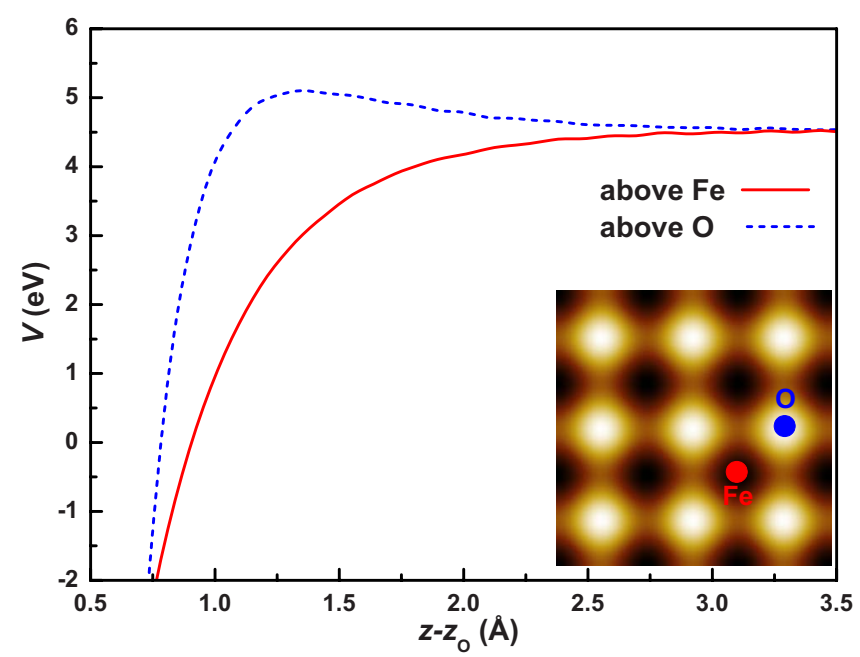

FIG. 3. (Color online) Electrostatic potential energy (including ionic cores) above the oxygen atoms (dashed blue line) and above the iron atoms (solid red line), along the [001] direction ( $z$ axis). Here, $z_{\mathrm{O}}$ represents the position of the oxygen atoms on the $z$ axis. Inset: simulated STM image for the $\mathrm{Fe}(001)-p(1 \times 1) \mathrm{O}$ surface at an average distance $\bar{z}=2 \AA$.

transfer occurs between the surface $\mathrm{Fe}$ and $\mathrm{O}$ atoms, the latter being negatively charged. As a consequence, the electrostatic potential acting on the electrons outside the surface is lower on top of $\mathrm{Fe}$ than on top of $\mathrm{O}$ atoms (solid and dashed lines in Fig. 3, respectively). We remark that such a potential has been evaluated by considering the actual optimized positions of both $\mathrm{O}$ and $\mathrm{Fe}$ atoms at the surface. The difference in the potential suggests that the electronic wave functions decay in the vacuum ( $z$ direction) more slowly when moving outward above the position of the iron atoms, compared to moving above the position of the oxygen atoms (see the reference spots in the inset of Fig. 3), therefore leading to the reversed corrugation at large distances. This qualitative and physically sound explanation is however not fully reproduced by our analysis based on DFT (see below).

Numerical simulations of the STM images have been performed at an average distance from the oxygen layer of about $2 \AA$. The use of either positive or negative small biases provided qualitatively identical images (see also Sec. II and Ref. 20). The results are equivalent to those obtained in our previous investigation ${ }^{24}$ and are shown in the inset of Fig. 3. At this distance the geometric contribution prevails and the bright spots correspond to the protruding oxygen atoms. This result should be compared with experimental data measured at high currents, hence sampling the surface at short distances.

To simulate the image at larger distances, an increased computational effort would be required (mainly, a larger basis set and a thicker slab). In principle, aiming to obtain the greatest deal of information from theoretical computations, this could still be afforded thanks to the small unit cell of the $p(1 \times 1)$ structure. On the other hand, the reliability of simulated images in such situations is limited by the fact that the exchange-correlation contribution to the total KS potential has been approximated by GGA, which is unsuited to capture the proper long-distance behavior. The corresponding 


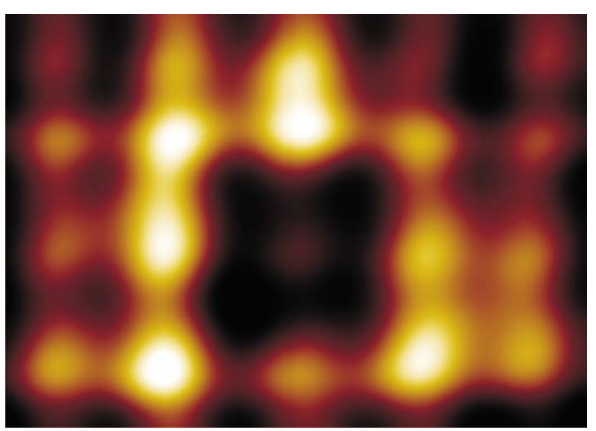

FIG. 4. (Color online) Blow up of the defect seen in the STM image of Fig. 1 with set point $V_{b}=0.1 \mathrm{~V}$ and $I=5 \mathrm{nA}$. The image area is about $1.5 \times 1.2 \mathrm{~nm}^{2}$

results are reported here for the sake of completeness only: we could span a distance range up to $8 \AA$, where the electron density of states is $10^{9}$ times smaller than the maximum value in the cell, and found that the simulated corrugation monotonically goes to zero with increasing distance, without ever reverting.

Finally, it is worth reminding that we do not consider the effects of the tip in the image formation. Nevertheless, we emphasize that the series of images shown in Fig. 1 could be repeatedly obtained, without significant modifications, by increasing or decreasing the tip-sample distance across the two atomic corrugation regimes. We can thus exclude that the corrugation reversal is due to a sudden irreversible alteration of the tip.

\section{B. Defect characterization}

We take now a closer look at the defect chosen as a reference for the investigation of the corrugation reversal, with the aim of understanding if our experimental and theoretical approaches allow to infer its nature. The topographic characteristics of this defect are best appreciated in the image taken at $V_{b}=0.1 \mathrm{~V}$ and $I=5 \mathrm{nA}$. On account of the previous analysis, we assume to be close enough to the surface to have the geometrical contributions dominating over the electronic ones in determining the topographic image of the surface, as seen by STM. Therefore, the protrusions seen on the image, excluding the defect region, are assigned to oxygen atoms.

In order to simulate the defective surface, we make the assumption that the defect consists of an oxygen vacancy. This choice is supported by an inspection of Fig. 4, which shows a blow up of the defect site and of its first neighbors, and by considering the surface preparation conditions mentioned above and the short tip-sample distance (high tunneling current regime). The defect, in particular, is centered on an oxygen site, involves a limited region, and presents a fourfold symmetry. Moreover we observe, by comparing different images of the same defect taken in the high current set point regime, and always making reference to the center of the defect, that both the first- and the second-neighbor protrusions are brighter than all of the other protrusions seen in the image.

In the simulation we have thus removed an oxygen atom from the perfect $p(1 \times 1)$ surface, on both sides of the slab,
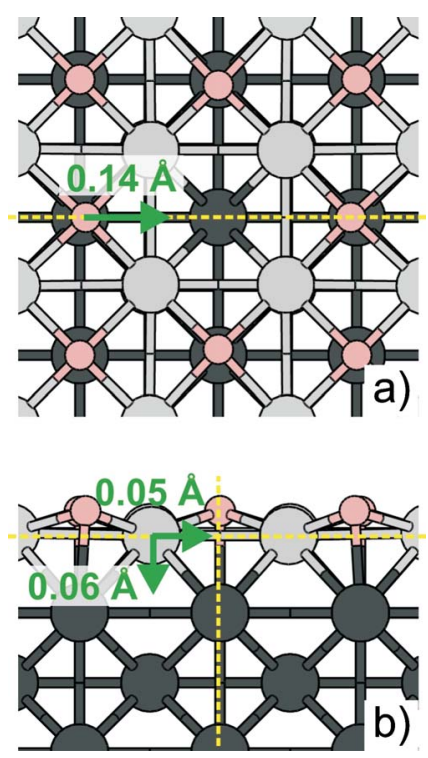

FIG. 5. (Color online) (a) Top view and (b) side view of the relaxed defective surface. The small circles represent the $\mathrm{O}$ atoms; the large circles represent the Fe atoms, both in the first layer (light gray) and in the layers below (dark gray). The arrows indicate the atom displacement, along the directions of the dashed lines, upon relaxation (see text).

and relaxed the structure in a $(4 \times 4)$ surface unit cell. Upon removal of the central oxygen atom, the four first-neighbors oxygen atoms relax in-plane toward the vacancy by $0.14 \AA$, with a negligible vertical displacement. The in-plane relaxation of the four in-plane first-neighbors Fe atoms amounts to $0.05 \AA$ toward the vacancy and $0.06 \AA$ in the vertical direction, reducing the separation from the inner Fe layer. The total energy of this relaxed surface is $0.26 \mathrm{eV}$ lower with respect to the unrelaxed one. A snapshot of the geometric configuration is reported in Fig. 5.

Using this configuration we computed the STM image as described in Sec. II; the result is reported in Fig. 6. The average distance between the chosen isosurface and the oxygen layer is about $2 \AA$. This distance must be related to a measure obtained in a high current regime, as that reported in

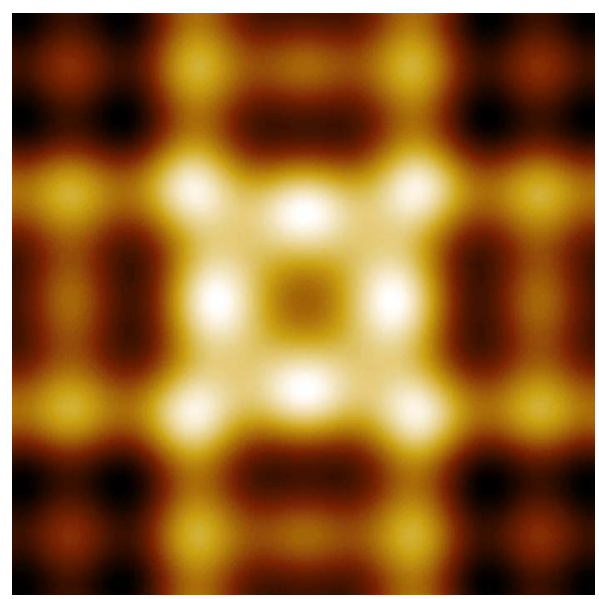

FIG. 6. (Color online) Simulated STM image for the relaxed defective surface. 
Fig. 4. The main feature of the experimental results, i.e., the increased brightness of the $\mathrm{O}$ atoms close to the vacancy, is indeed nicely reproduced. It is worth to notice that, in the central region, the absence of the oxygen atom should require a shorter tip-surface distance to keep a fixed current. Possibly, the weak bright spot at the center of the defect shown in Fig. 4, which is missing in the simulated image shown in Fig. 6, could be due to the influence of the scanning tip. The overall good agreement between the two set of results suggests to actually identify the measured defect as due to an oxygen vacancy.

\section{DISCUSSION}

On the basis of the experimental and theoretical results described in the previous sections, we now consider and discuss three main factors that can be taken into account to understand how the STM images of both the perfect and the defective $\mathrm{Fe}(001)-p(1 \times 1) \mathrm{O}$ surface take shape.

The first and more intuitive factor is the geometrical configuration, which is often invoked to identify the brightest spots with the atoms located in the highest position with respect to the surface. In the present system, however, the presence of two atomic species suggests that this direct interpretation can easily be misleading. Moreover we have seen, when analyzing an oxygen vacancy, that the $\mathrm{O}$ atoms close to the defect, which experimentally appear to be brighter than the others in the image, are actually at the same distance from the surface as any of the others. We must therefore exclude that the geometrical arrangement of the oxygen atoms can be considered as the main reason for the experimental evidences.

A second contribution to be reckoned in is the charge distribution (or, more precisely, the local density of states in the proper energy interval close to $E_{\mathrm{F}}$ ) on the different atoms present at the surface. By performing a Löwdin charge analysis, ${ }^{27}$ we find that, in the perfect $\mathrm{Fe}(001)-p(1 \times 1) \mathrm{O}$ surface, each oxygen atom holds an additional electron captured from the underlying $\mathrm{Fe}$ atoms. Upon removal of an oxygen atom, such an electron is shared between the five firstneighbors Fe atoms (four in the surface plane and one in the first layer below) and only a small fraction of the charge is distributed on the other $\mathrm{O}$ atoms. Moreover, we do not find significant differences in the local density of states at the atoms nearest to the vacancy, when compared to those on the perfect $\mathrm{Fe}(001)-p(1 \times 1) \mathrm{O}$ surface. We conclude that the details of the charge distribution on the specific orbitals of the different species present at the surface, even if possibly modified upon the defect creation, have a negligible influence on the details of the image formation of the oxygen vacancy.

Finally, we discuss the role played by the decaying behavior toward the vacuum of the electronic wave functions involved, which is affected by the local potential barrier above the surface. A modification of such a barrier, caused either by oxygen adsorption, when passing from the pristine $\mathrm{Fe}(001)$ surface to the $\mathrm{Fe}(001)-p(1 \times 1) \mathrm{O}$ surface, or by oxygen removal, when the vacancy is created, can in fact be responsible of either promoting or hindering the tunneling process

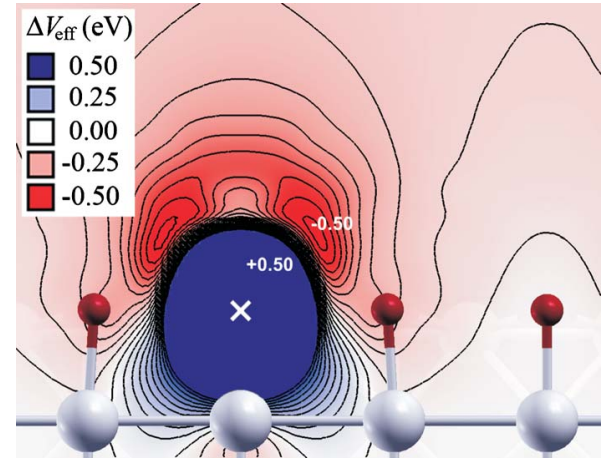

FIG. 7. (Color online) Difference in the effective KS potential between the $\mathrm{Fe}(001)-p(1 \times 1) \mathrm{O}$ surface with the vacancy (i.e., in absence of the $\mathrm{O}$ potential, which is strongly negative close to the nucleus) and the same surface without the vacancy (indicated by a white cross). The small spheres represent the $\mathrm{O}$ atoms; the large spheres represent the $\mathrm{Fe}$ atoms in the first subsurface layer.

to the STM tip. The contribution of the local potential barrier remains the only one that can play a role in the corrugation reversal previously discussed for the $\mathrm{Fe}(001)-p(1 \times 1) \mathrm{O}$ surface. We remark that possible alterations of the surface potential barrier might in principle be induced also by the interaction with the STM tip, ${ }^{8}$ whose effect has not been considered in our analysis. Nevertheless we note that, to explain the observed findings, such tip induced alterations should be more effective when the tip is farther from the surface than when it is closer, which seems a counterintuitive result.

It is therefore interesting to evaluate the variation of the potential acting on the electrons due to the creation of the $\mathrm{O}$ vacancy. To this purpose, we report in Fig. 7 the difference in the KS potential between the defective surface discussed in Sec. III B and the one obtained by reintroducing the removed $\mathrm{O}$ atom into the vacancy site. The dark shaded region (blue in the color version, $\Delta V_{\text {eff }} \geq+0.5 \mathrm{eV}$ ) around the vacancy site (i.e., in a region around the defect position of smaller extent than the atomic size) corresponds to an increase of the potential in presence of the vacancy, mostly due to the missing potential of the removed $\mathrm{O}$ atom, which is strongly negative close to the nucleus. On the contrary, in the less dark (red in the color version, $\Delta V_{\text {eff }} \approx-0.5 \mathrm{eV}$ ) symmetric lobes above the vacancy region the potential is lower and consequently the decay of the wave functions is slower. To be more intuitive, the latter regions represent preferential channels for the tunneling process. Thanks to the presence of these channels, the electron states on the $\mathrm{O}$ atoms closest to the vacancy can more easily extend away from the surface, therefore resulting in the increased brightness of the firstneighbor $\mathrm{O}$ atoms. On the other hand, in the vacancy position, the STM tip would be forced to come closer to the surface to succeed in coupling its electron states with those of the sample, there mainly located in the subsurface $\mathrm{Fe}$ atom.

As a final remark, we underline that one should not deduce, from the previous considerations, that the atomic configuration is irrelevant in determining the STM image formation, even though no significant $\mathrm{O}$ vertical displacements are 
found upon the relaxation following the creation of the $\mathrm{O}$ vacancy. In fact we have verified, by further simulations, that the STM image obtained with an unrelaxed atomic configuration (not shown here) is much different from the one shown in Fig. 6. Such an image actually shows a very pronounced bright spot on top of the vacancy, which overwhelms the other features, and does not reproduce the experimental evidence.

\section{CONCLUSIONS}

We have reported on an atomically resolved investigation of the Fe(001)- $p(1 \times 1) \mathrm{O}$ surface by STM. In particular, we have focused our attention on a corrugation reversal between oxygen only and iron only atoms seen on the surface, as a function of the tip-sample distance. Comparison between experimental images and first-principles calculations suggests that the observed phenomenology is due to the different local potential barrier on top of either $\mathrm{O}$ or $\mathrm{Fe}$ surface atoms. Based on this conclusion, we have analyzed an oxygen vacancy created on the surface, and have been able to produce a simulated image in fairly good agreement with the measurements. We believe that our study can represent a suitable general approach for the interpretation of STM images of oxidized metal surfaces where, in particular, the geometrical structure cannot be a support for the understanding of the experimental observations.

\section{ACKNOWLEDGMENTS}

The authors acknowledge financial support by Fondazione Cariplo through the project IMMAGINA (Grant No. Rif. 20085.2412) and by MIUR through the PRIN Project No. 2007 CMLFY2. *alberto.brambilla@polimi.it

${ }^{1}$ M. Finazzi, L. Duò, and F. Ciccacci, Surf. Sci. Rep. 64, 139 (2009).

${ }^{2}$ G. Pacchioni, ChemPhysChem 4, 1041 (2003).

${ }^{3}$ F. Calleja, A. Arnau, J. J. Hinarejos, A. L. Vázquez de Parga, W. A. Hofer, P. M. Echenique, and R. Miranda, Phys. Rev. Lett. 92, 206101 (2004).

${ }^{4}$ R. Schaub, E. Wahlstrom, A. Ronnau, E. Lagsgaard, I. Stensgaard, and F. Besenbacher, Science 299, 377 (2003).

${ }^{5}$ M. R. Castell, P. L. Wincott, N. G. Condon, C. Muggelberg, G. Thornton, S. L. Dudarev, A. P. Sutton, and G. A. D. Briggs, Phys. Rev. B 55, 7859 (1997).

${ }^{6}$ D. P. Woodruff, Curr. Opin. Solid State Mater. Sci. 7, 75 (2003).

${ }^{7}$ F. Besenbacher, Rep. Prog. Phys. 59, 1737 (1996).

${ }^{8}$ W. A. Hofer, J. Redinger, A. Biedermann, and P. Varga, Surf. Sci. 466, L795 (2000).

${ }^{9}$ L. Giordano, G. Pacchioni, J. Goniakowski, N. Nilius, E. D. L. Rienks, and H.-J. Freund, Phys. Rev. B 76, 075416 (2007).

${ }^{10}$ K. O. Legg, F. Jona, D. W. Jepsen, and P. M. Marcus, Phys. Rev. B 16, 5271 (1977).

${ }^{11}$ F. Jona and P. M. Marcus, Solid State Commun. 64, 667 (1987).

${ }^{12}$ R. L. Headrick, P. Konarski, S. M. Yalisove, and W. R. Graham, Phys. Rev. B 39, 5713 (1989).

${ }^{13}$ R. Bertacco, S. De Rossi, and F. Ciccacci, J. Vac. Sci. Technol. A 16, 2277 (1998).

${ }^{14}$ R. Bertacco, M. Merano, and F. Ciccacci, Appl. Phys. Lett. 72, 2050 (1998)
${ }^{15}$ F. Bisio, R. Moroni, M. Canepa, L. Mattera, R. Bertacco, and F. Ciccacci, Phys. Rev. Lett. 83, 4868 (1999).

${ }^{16}$ J. P. Perdew, K. Burke, and M. Ernzerhof, Phys. Rev. Lett. 77, 3865 (1996).

${ }^{17}$ D. Vanderbilt, Phys. Rev. B 41, 7892 (1990).

${ }^{18}$ P. Giannozzi, S. Baroni, N. Bonini, M. Calandra, R. Car, C. Cavazzoni, D. Ceresoli, G. L. Chiarotti, M. Cococcioni, I. Dabo et al., J. Phys.: Condens. Matter 21, 395502 (2009); http:// www.quantum-espresso.org

${ }^{19}$ H. J. Monkhorst and J. D. Pack, Phys. Rev. B 13, 5188 (1976).

${ }^{20} \mathrm{~A}$ moderately large bias allows one to obtain numerically stable results already for thin slabs. We have verified, for the $\mathrm{Fe}(001)-p(1 \times 1) \mathrm{O}$ surface modeled by a thicker (15 layers) slab, that qualitatively equivalent results are obtained when smaller (positive or negative) biases are applied.

${ }^{21}$ A. Selloni, P. Carnevali, E. Tosatti, and C. D. Chen, Phys. Rev. B 31, 2602 (1985).

${ }^{22}$ N. D. Lang, Phys. Rev. B 34, 5947 (1986).

${ }^{23}$ J. A. Stroscio, D. T. Pierce, A. Davies, R. J. Celotta, and M. Weinert, Phys. Rev. Lett. 75, 2960 (1995).

${ }^{24}$ F. Donati, P. Sessi, S. Achilli, A. Li Bassi, M. Passoni, C. S. Casari, C. E. Bottani, A. Brambilla, A. Picone, M. Finazzi, L. Duò, M. I. Trioni, and F. Ciccacci, Phys. Rev. B 79, 195430 (2009).

${ }^{25}$ P. Blonski, A. Kiejna, and J. Hafner, Surf. Sci. 590, 88 (2005).

${ }^{26}$ S. R. Chubb and W. E. Pickett, Phys. Rev. Lett. 58, 1248 (1987).

${ }^{27}$ P.-O. Löwdin, J. Chem. Phys. 18, 365 (1950). 